\title{
Gute Argumente, unhaltbare Schlussfolgerungen
}

\section{Daniele Zullinoa, Daniel Büche ${ }^{b}$, Sandro Cattacinc ${ }^{c}$, Thilo Beck ${ }^{d}$, Louise Penzenstadler}

${ }^{a}$ Prof. Dr. med., Service d'Addictologie, Hôpitaux Universitaires de Genève; ${ }^{b}$ Dr. med., Palliativzentrum, Kantonsspital St. Gallen; ${ }^{c}$ Prof., Département de



Der Artikel des Kollegen Barben wirft einerseits absolut berechtigte Fragen auf. Er ist in seinem logischen Aufbau allerdings absolut unhaltbar und dabei leider beispielhaft für eine mittlerweile alles andere als wohlreflektierte Debatte um die Möglichkeiten einer Reform der Regulation des Cannabismarktes. Der Artikel will gegen eine Lockerung des derzeitigen Regimes argumentieren. Wie im Folgenden aufgezeigt werden soll, kann er dies allerdings aufgrund verschiedener formallogischer Fehler nicht leisten. Es soll hier, unter anderem im Namen der Swiss Society of Addiction Medicine (SSAM) und des Collège Romand de Médecine d'Addiction (COROMA), die Argumentation des Artikels hinterfragt werden.

Was ist also falsch an diesen Argumenten? Aus Sicht der Argumentationstheorie ist ein Argument eine Aufeinanderfolge von Aussagen, die aus Prämissen (Voraussetzungen) und einer Schlussfolgerung (Konklusion) besteht. Damit ein Argument gültig ist, sollen die Prämissen die Konklusion begründen.

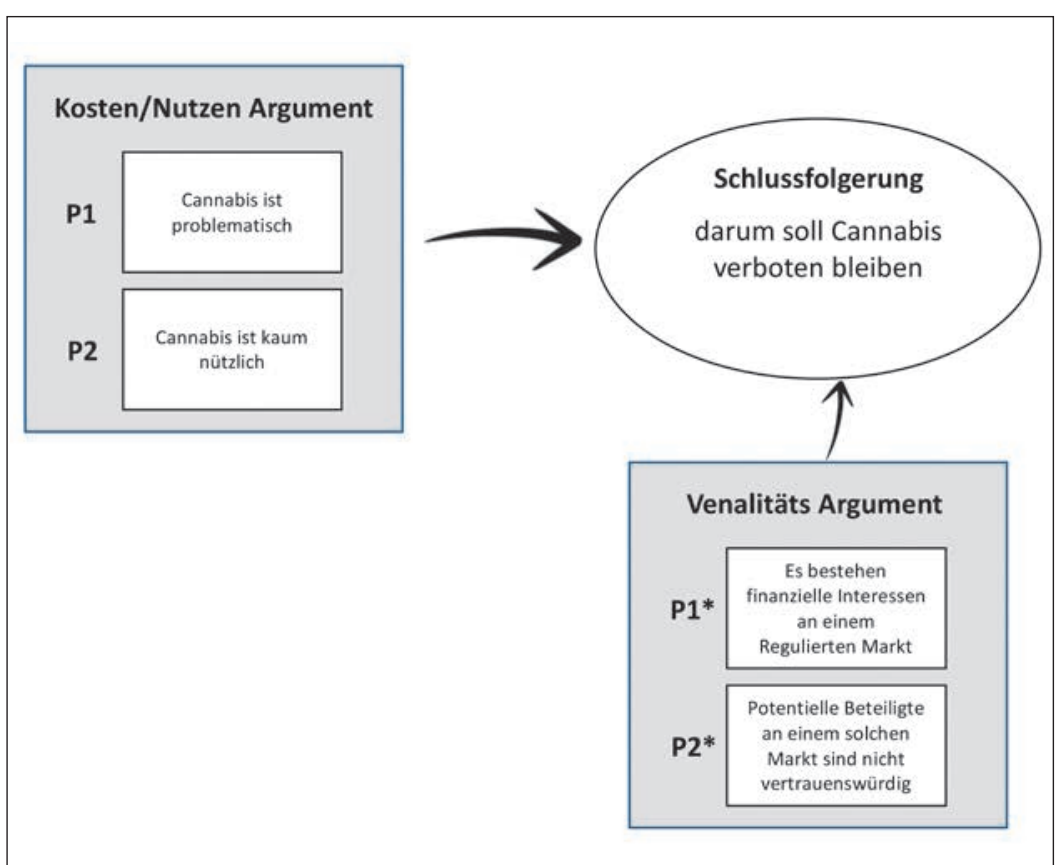

Abbildung 1: Trugschlüsse.
Der Artikel von Herrn Barben verwendet formal ganz eindeutig ein Deduktives Argument, nur eben in der präsentierten Form kein gültiges Argument. Einerseits sind gewisse aufgeführte Prämissen selber unbegründet, andererseits lassen sich auch bei eventuellem $\mathrm{Zu}$ lassen dieser Prämissen die Schlussfolgerungen nicht nach den Gesetzen der Logik ziehen. Bei der im Artikel gezogenen Schlussfolgerung handelt es sich formallogisch um einen groben Fehlschluss. Sollte das ungültige Argument sogar mit Absicht verwendet worden sein, würde es sich dann definitionsgemäss gar um einen Sophismus (Scheinargument) handeln.

Bei der im Artikel gezogenen Schlussfolgerung handelt es sich formallogisch um einen groben Fehlschluss.

Die Argumentation im Artikel von Professor Barben ist in Abbildung 1 schematisch wiedergegeben. Es lassen sich an und für sich zwei unterschiedliche Argumentationsstränge erkennen, die wir in der Folge kurz getrennt auf ihre Gültigkeit untersuchen.

\section{Das Kosten/Nutzen-Argument}

Gemäss diesem Argument sollten also den durch Cannabis bedingten Risiken (P1) durch eine Beibehaltung der derzeitigen Regulierung (= Prohibition der Substanz) entgegengewirkt werden (S), da zudem Cannabis kaum therapeutisch ist (P2). Dies ist an und für sich das in der Prohibitions/Regulations-Debatte klassischerweise diskutierte Argument. Damit dieses Argument valide ist, müssten wie oben präzisiert (a) die Prämissen wahr sein (von der eventuellen Gegenseite als wahr akzeptiert werden) und sich (b) aus der Verknüpfung der Prämissen die Schlussfolgerung zwingend ergeben. Die Argumentation Herrn Barbens lässt sich hierbei sowohl auf den Wahrheitsgehalt der Prämissen als auch in Bezug auf den logischen Zusammenhalt zwischen Prämissen und Schlussfolgerung untersuchen.

Wir wollen in diesem Rahmen nur oberflächlich auf die präsentierten Prämissen eingehen. 
Ad (P1): Es dürfte unbestritten sein, dass der Cannabiskonsum, und dabei insbesondere das Rauchen von Cannabis, auf individuellem und volksgesundheitlichem Niveau negative Auswirkungen haben kann. Dabei stellen Jugendliche eine besonders vulnerable Gruppe dar, und ein früher Konsumbeginn erhöht sowohl das Risiko einer Suchtentwicklung als auch jenes von Folgeerscheinungen. Es soll hier nicht auf die Gewichtung dieses Risikos (z.B. seine Relevanz im Vergleich zu anderen Risiken) eingegangen werden. Wir werden um der Diskussion willen also (P1) als wahr zulassen. Ad (P2): Es wird im Artikel wiederholt deklariert, dass der medizinische Nutzen von Cannabis gering sei, eine Behauptung, die kaum durch die zitierten Literaturstellen belegbar ist (Referenzen 4-7). Wir werden jedoch auch (P2) der Form halber als wahr annehmen.

Stellt sich also nach Akzeptanz der Prämissen die Frage, ob sich aus der Verknüpfung von (P1) und (P2) die Schlussfolgerung ( $\mathrm{S}=$ Prohibition) ziehen lässt. Wir könnten das Argument auch umformulieren: «Cannabis ist ein Problem, darum gehört es verboten.» Hierbei wird ersichtlich, dass mit der Formulierung suggeriert wird, dass das Verbot (S) die einzige Lösung des Problems ist. Dies lässt sich allerdings nicht aus den gegebenen Prämissen ableiten. In Wirklichkeit wäre die Aussage «Prohibition ist eine/die Lösung» eine weitere Prämisse, die zuvor noch auf ihren Wahrheitsgehalt untersucht werden müsste. Dies ist in Abbildung 2 als P3 schematisch dargestellt.

Dass (P3) nicht als wahr angenommen werden kann, ist durch die eigene Beschreibung Herrn Barbens einer steigenden Prävalenz der Cannabisraucher, der THCKonzentrationen und der hiermit verbundenen Risi-

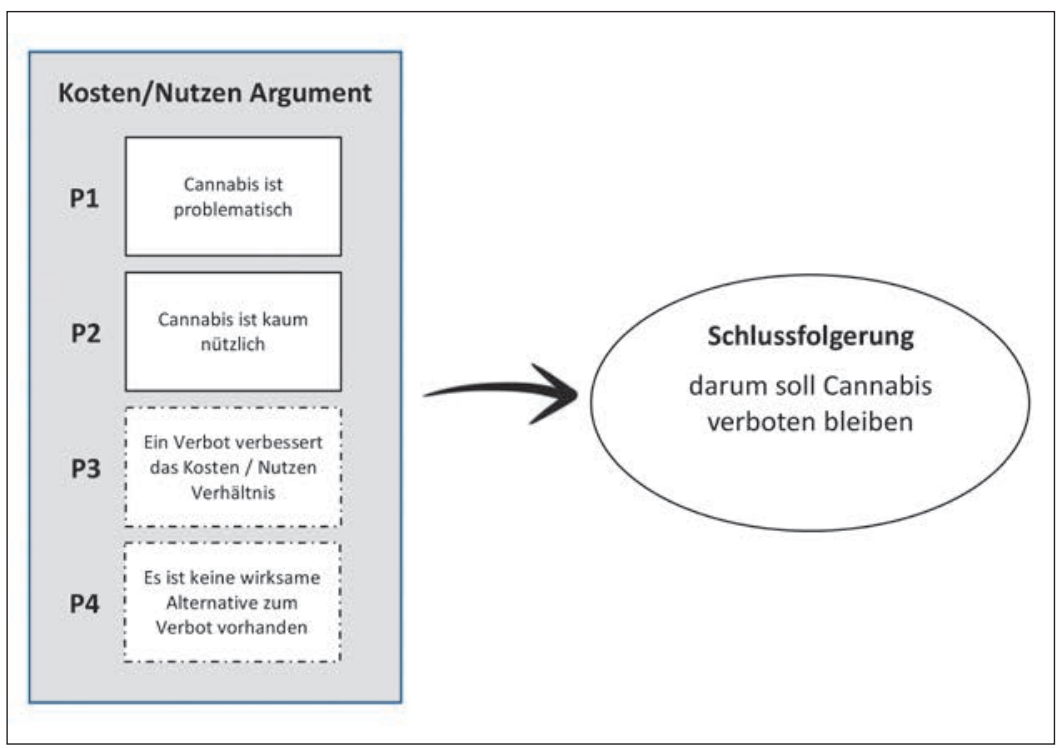

Abbildung 2: Vervollständigtes Argument. ken belegt ... all dies bei bestehender Prohibition. Das Risiko welches durch die Beibehaltung der Prohibition verhindert werden soll, ist bereits realisiert!

In einem liberalen Rechtsstaat müssen freiheitseinschränkende Massnahmen grundsätzlich einem Zweck dienen (Kriterium der Wirksamkeit) und dieser Zweck darf anders nicht erreichbar sein (Kriterium der

\section{Wer freien Staatsbürgern etwas verbieten} will, muss die Evidenzen erbringen und nicht umgekehrt.

Notwendigkeit). Wäre (P3) also richtig, müsste immer noch (P4), das Fehlen einer Alternative zum Verbot, belegt werden. Die Beweislast liegt hierbei wohlgemerkt grundsätzlich beim Prohibitionisten und nicht bei der Gegenseite. Wer freien Staatsbürgern etwas verbieten will, muss die Evidenzen erbringen und nicht umgekehrt. Diese Grundsätze finden sich übrigens auch im Artikel 36 (Einschränkungen von Grundrechten) der Schweizer Bundesverfassung. Was im Falle von Herrn Barbens Argumentation also fehlt, ist das Verbindungsstück (P3 und P4) zwischen der Prämisse "Cannabis ist ein Risiko» und der Schlussfolgerung «Cannabis gehört verboten».

Es handelt sich beim von Herrn Barben vorgebrachten Kosten/Nutzen-Argument um einen klassischen Nonsequitur-Fehlschluss, eine Schlussfolgerung, die nicht aus den Voraussetzungen folgt, unabhängig davon, ob die Voraussetzungen zutreffen oder nicht. Ist dies unbeabsichtigt erfolgt, ist es zumindest ungeschickt. War eine Absicht dahinter, handelt es sich um Sophisterei, die zwar als politisch clever gelten mag, aber intellektuell unredlich ist.

Es könnte nun allerdings eingewandt werden, dass die Prohibition zwar gesundheitspolitisch relativ unwirksam ist, eine Reform des Regimes allerdings unvorhersehbare, noch negativere Auswirkungen haben könnte. Dass dies den Prinzipien liberaler Rechtsstaatlichkeit zuwiderläuft, soll nicht noch einmal erörtert werden. Es soll nur auf die in der Zwischenzeit überwältigenden Evidenzen aus den USA verwiesen werden (z.B. die Daten des National Survey on Drug Use and Health), die solchen Befürchtungen klar die Berechtigung entziehen. Und um es nochmals klar zu machen: Die Beweislast wäre an und für sich bei den Prohibitionisten.

\section{Das Venalitätsargument}

Die eigentliche Originalität des Artikels von Herrn Barben liegt in den eindrücklich dokumentierten Textstellen bezüglich der finanziellen Interessen- 


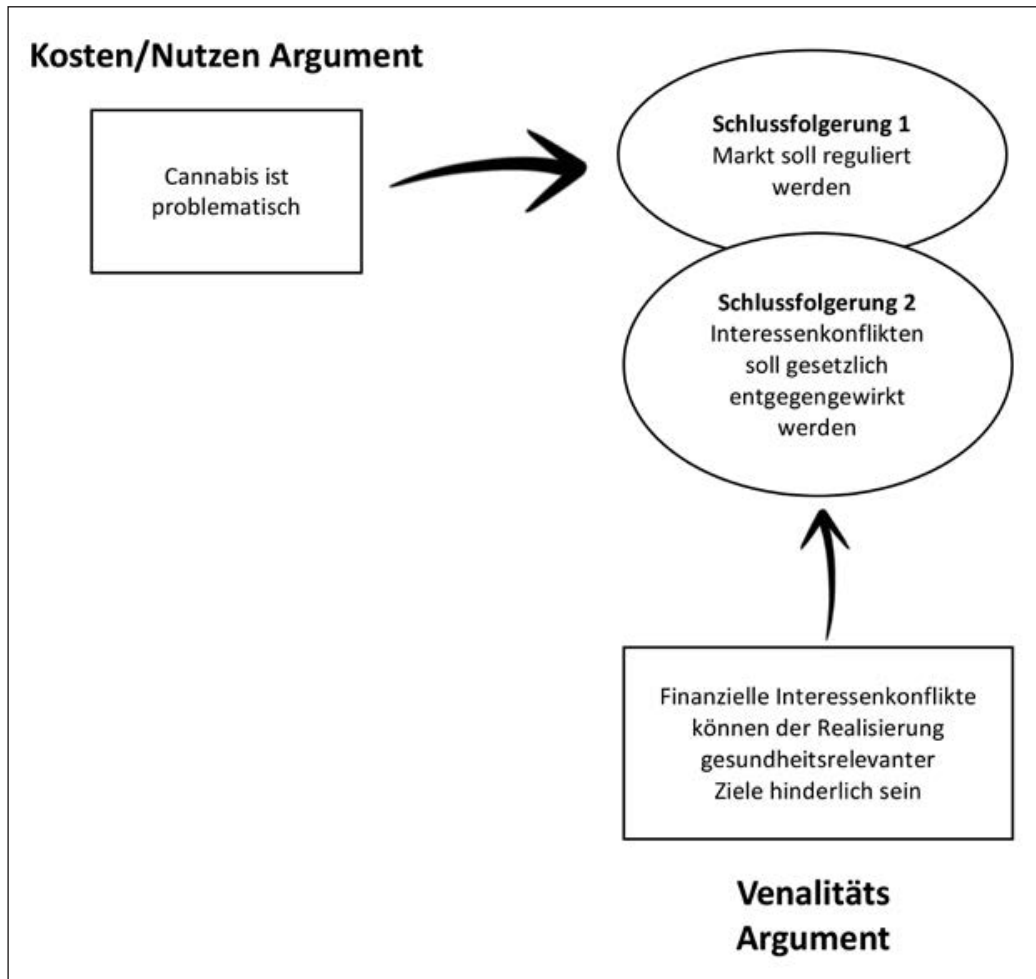

Abbildung 3: Logisch gültige Argumente.

konflikte, welche sich aus einer eventuellen Reform der Cannabisregulierung ergeben könnten. Wir teilen Herrn Barbens Bedenken hierbei vollumfänglich.

Allerdings sind auch die in diesem Zusammenhang formulierten Prämissen (P3 und P4 in Abb. 1) kein logischer Grund für die Schlussfolgerung «Prohibition». Und zwar aus mindestens zwei Gründen.

Es wird nicht ganz ersichtlich, inwiefern der sich ausbreitende Markt von CBD-Cannabis die Beibehaltung der Prohibition begründen sollte.

Erstens, sie tragen nicht zur Validität des Kosten/Nutzen-Arguments bei, da jenes, wie oben dargelegt, ohnedies schon nicht valide ist. Das Hinzufügen des Venalitätsarguments entspricht in diesem Fall einem sogenannte Red Herring-Sophismus. Der Begriff Red Herring (auch falsche Fährte oder Verschiebung der Streitfrage) bezeichnet eine Gruppe von Scheinbeweisen, die interessante, scheinbar plausible, aber letztlich für die Schlussfolgerung irrelevante Prämissen einbringen. Auf den vorliegenden Fall bezogen: Die Prohibition bleibt auch dann unwirksam (P3 in Abb. 1), wenn das Venalitätsargument eingebracht wird. Letzteres ist unter diesen Umständen also irrelevant.
Der Text ist zudem mit zahlreichen weiteren Red Herrings gespickt. Es wird unter anderem nicht ganz ersichtlich, inwiefern der sich ausbreitende Markt von CBD-Cannabis die Beibehaltung der Prohibition begründen sollte. Die Tatsache, dass bereits an die 600 Firmen an diesem Markt beteiligt sind und dass dieses Geschäft 15 Millionen an Steuereinnahmen generiert hat, scheint erneut als Argument für eine Prohibition zu dienen, ohne dass der Autor erklärt, inwiefern dies an und für sich problematisch sei. Auch ist der Hinweis auf das Aufkommen synthetischer Cannabinoide und der E-Zigaretten mit den damit verbundenen Risiken ein weiterer Ablenker, der formallogisch in keinem Zusammenhang mit der Schlussfolgerung steht.

Zwar ist tatsächlich ziemlich gut belegt, dass die Zulassung von Cannabis für medizinische Zwecke nur als Vorstufe für die spätere vollständige Legalisierung benutzt wurde. Aber erneut ist bei genauerem Hinsehen ein logischer Zusammenhang mit der geforderten Schlussfolgerung nicht ersichtlich. Es handelt sich hierbei, gleich wie bei der Bemerkung, dass einer der ProReform-Lobbyisten Vermögen als Finanzspekulant gemacht hat, um eine Sonderform des Red Herring, das ad personam-Manöver. Ein ad personam geschieht in der Absicht, die Position der Gegenseite in Misskredit zu bringen und eine echte Diskussion (verbessert die Prohibition das Kosten/Nutzen-Verhältnis?) zu vermeiden.

Zweitens ist das Venalitätsargument auch isoliert für sich genommen ein Non-sequitur-Fehlschluss. Es müssten weitere Prämissen hinzugefügt werden, welche die Verbindung zwischen den finanziellen Interessenkonflikten und der Prohibition aufzeigten. Eine solche Prämisse könnte sein: «Es soll niemand Geld mit Cannabis verdienen.» In diesem Fall wäre die Kombination mit dem Kosten/Nutzen-Argument gar nicht mehr nötig. Wer eine solche Prämisse vorbringt, müsste sich allerdings den Vorwurf gefallen lassen, den Verdienst im illegalen Markt zu akzeptieren, aber nicht jenen in einem staatlich regulierten.

\section{Die Argumente unterstützen einen staatlich regulierten Markt}

Dass die Aussicht auf einen legal regulierten Markt finanzielle Begehrlichkeiten weckt, und diese gesundheitsrelevant sein können, ist durch den Artikel von Herrn Professor Barben deutlich hervorgehoben worden. Dass aber die Steuereinnahmen die Folgekosten nicht decken werden, ist eine Behauptung, deren Beweislast an und für sich beim Autor läge. Dass es bei 
der Tabakepidemie der Fall war (und ist), darf nicht genügen. Da im Fall eines eventuellen regulierten Cannabismarktes im Voraus (a priori) reguliert würde, kann nicht bereits automatisch auf eine gleich mangelhaft gestaltete Steuerpolitik geschlossen werden, wie es bezüglich des a posteriori geregelten Tabakmarktes der Fall war.

Jedwede Regelung des Cannabismarktes sollte eine strikte Kontrolle der finanziellen Interessenkonflikte beinhalten.

Dass die Tabakindustrie in Bezug auf die von ihr ausgehenden Risiken absolut nicht vertrauenswürdig ist, muss nicht weiter diskutiert werden. Sicherlich besteht auch bei neu auftretenden, nicht mit der Tabakindustrie gekoppelten Unternehmen das Risiko, das unheilvolle Geschäftsgebaren der Tabakproduzenten zu übernehmen.

Das Venalitätsargument kann, wie oben belegt, eine volksgesundheitlich motivierte Prohibition logischerweise nicht begründen. Wir gehen aber mit Kollege Barben darin einig, dass jedwede Regelung des Cannabismarktes eine strikte Kontrolle der finanziellen Interessenkonflikte beinhalten sollte. Das Interesse der Tabakindustrie für einen sich in die Legalität verschiebenden Markt dürfte tatsächlich eines der grössten Risiken sein. Es braucht wohl kaum noch Evidenzen für die Rücksichtslosigkeit von deren Repräsentanten. Ein grundsätzlicher und nachdrücklich überprüfter Ausschluss der Tabakindustrie erscheint als eine der Grundbedingungen, sollte ein regulierter Cannabismarkt die in ihn gesetzten volksgesundheitlichen Hoffnungen erfüllen können. Eine klare Trennung von den Märkten für weitere Suchtmittel (Alkohol, Glücksspiel, Gaming etc.) sollte ebenfalls ernsthaft in Betracht gezogen werden.

\section{Schlussfolgerung}

Wir sind mit Kollege Barben einig, dass ein weitverbreiteter Cannabiskonsum ein volksgesundheitliches Risiko darstellt, dem entgegengewirkt werden muss. Die Evidenzen der Überlegenheit eines staatlich straff geregelten Marktes über den Ansatz eines generellen Verbotes dürften dabei heute vor allem von uns Fachpersonen eigentlich nicht mehr ignoriert werden. In diesem Zusammenhang muss ökonomischen Interessenkonflikten unbestrittenerweise ein besonderes Augenmerk zukommen. Die Argumentationen Herrn Barbens sind also insgesamt, wenn seine Prämissen adäquat ergänzt werden, bestens geeignet, eine Regulierung des Cannabismarktes zu unterstützen. Das grosse Verdienst seines Artikels ist in diesem Fall, auf mögliche Fallstricke hingewiesen zu haben.

Literatur

1 Barben J. Cannabis-Legalisierung - wer profitiert davon? Schweiz Ärzteztg. 2018;99(48):1710-2.

\section{Dieser Artikel wird mitgetragen von folgenden Fachpersonen}

PD Dr. med. Sophia Achab, Service d'Addictologie, Hôpitaux Universitaires de Genève; Prof. Dr. med. Barbara Broers, Service de Médecine de Premier Recours, Hôpitaux Universitaires de Genève; Med. pract. Gerard Calzada, Service d'Addictologie, Hôpitaux Universitaires de Genève; Dr. med. Robert Hämmig, Bern; Dr. med. André Kuntz, Centre cantonal d'addictologie, Réseau Fribourgeois de Santé Mentale; Dr. med. Jeorge Riesen, Courlevon; Jean-Felix Savary, Groupement Romand d'Etudes des Addictions; Dr. med. Olivier Simon, Service de psychiatrie communautaire, Centre Hospitalier Universitaire Vaudois; PD Dr. med. Gabriel Thorens, Service d'Addictologie, Hôpitaux Universitaires de Genève; Dr. med. Stergios Tsartsalis, Service de Psychiatrie Adulte, Hôpitaux Universitaires de Genève; PD Dr. med. Marc Vogel, Universitäre Psychiatrische Kliniken Basel; Prof. Dr. med. Marc Walter, Universitäre Psychiatrische Kliniken Basel 